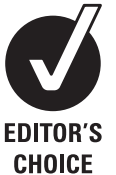

CHOICE
${ }^{1}$ Department of Social Medicine, School of Public Health, Harbin Medical University, Harbin, Heilongjiang, People's Republic of China

${ }^{2}$ School of Public Health, La Trobe University, Victoria, Australia

${ }^{3}$ Heilongjiang Provincial Center for STD Prevention and Control, Harbin, Heilongjiang, People's Republic of China

${ }^{4}$ Heilongjiang Provincial Center for Tuberculosis Prevention and Control, Harbin, Heilongjiang,

People's Republic of China ${ }^{5}$ School of Public Health, Peking University, Beijing, People's Republic of China

\section{Correspondence to}

Professor Qunhong Wu, School of Public Health, Harbin Medical University, 157 Baojian Road, Nangang District, Harbin, Heilongjiang 10081, People's Republic of China; wuqunhong@163.com

Libo Liang, Lijun Gao, Yanguang Xie contributed equally to this article.

Received 12 February 2011 Accepted 4 January 2012 Published Online First 8 March 2012

\title{
Factors contributing to the high prevalence of multidrug-resistant tuberculosis: a study from China
}

\author{
Libo Liang, ${ }^{1}$ Qunhong Wu, ${ }^{1}$ Lijun Gao, ${ }^{1}$ Yanhua Hao, ${ }^{1}$ Chaojie Liu, ${ }^{2}$ Yanguang Xie, ${ }^{3}$ \\ Hong Sun, ${ }^{1}$ Xinglu Yan, ${ }^{4}$ Fabin Li, ${ }^{4}$ Honghai Li, ${ }^{4}$ Hongxia Fang, ${ }^{4,5}$ Ning Ning, ${ }^{1}$ Yu Cui, ${ }^{1}$ \\ Liyuan $\operatorname{Han}^{1}$
}

\section{ABSTRACT}

Background The rapid spread of multidrug-resistant tuberculosis (MDR-TB) has attracted global concerns. This study aimed to identify factors contributing to the high prevalence of MDR-TB in China's Heilongjiang province.

Methods A cross-sectional survey following the WHO/ International Union Against Tuberculosis and Lung Disease guidelines was conducted with consecutive recruitment of patients with TB in 30 counties selected at random in Heilongjiang in 2004. A total of 1995 patients were tested for MDR-TB. Factors associated with MDR-TB were identified through multilevel models and traditional logistic regression analysis, along with in-depth interviews with nine patients, five healthcare managers and four doctors.

Results 241 patients (12\%) were identified with MDRTB. The retreatment patients were 5.48 times $195 \% \mathrm{Cl}$ 4.04 to 7.44) more likely to have MDR-TB than newly diagnosed patients. The patients who were treated with isoniazid and rifampin for $>180$ days were 4.82 times $(95 \% \mathrm{Cl} 2.97$ to 7.81$)$ more likely to develop MDR-TB than those treated $<180$ days. Age and delay in initiating TB treatment were associated with MDR-TB. Financial burden, poor knowledge and side effects of TB treatment were perceived by the interviewees as influencing factors. Lack of coordination of services, unsatisfactory supervision of treatment and infection control jeopardised the control of MDR-TB.

Conclusions Inappropriate treatment is the most important influencing factor of MDR-TB. Increasing people's awareness of TB, early detection and appropriate treatment of patients with TB should become a priority, which requires strong commitment and collaboration among health organisations and greater compliance with TB treatment guidelines by service providers and patients.

\section{INTRODUCTION}

The rapid expansion of multidrug-resistant tuberculosis (MDR-TB; ie, resistance to isoniazid and rifampin) has jeopardised global control of $\mathrm{TB}{ }^{1}$ MDR-TB exacerbates the existing grim $\mathrm{TB}$ epidemic, poses a great threat to global TB control and incurs a huge burden on developing countries due to its difficult, expensive, less effective and toxic treatment. ${ }^{12}$

China carries the greatest burden of MDR-TB The number of MDR-TB cases is the highest in the

\section{Key messages}

What is the key question?

- The rapid spread of multidrug-resistant tuberculosis (MDR-TB) has caused serious concerns in China.

What is the bottom line?

- The underlying causes of MDR-TB are still not clear.

\section{Why read on?}

- This study aimed to identify the factors contributing to the high prevalence of MDR-TB in China's Heilongjiang province.

world despite having the second highest number of TB cases. ${ }^{3}{ }^{4}$ It has been estimated that about 100000 new MDR-TB cases emerge every year in China. ${ }^{3}$ Alarmed by the serious MDR-TB epidemic, the Chinese government has commenced action. ${ }^{5}$ Joint TB control programmes with international partners have been initiated but progress has been far from satisfactory. ${ }^{6}$ The prevalence of MDR-TB has continued to rise. ${ }^{7}$

It is critical to identify the underlying reasons for MDR-TB so that more feasible and cost-effective approaches can be developed to control MDR-TB. Studies in some countries have identified retreatment, alcohol abuse, age, and immigrant status as factors influencing MDR-TB. ${ }^{8-11}$

This study was undertaken in Heilongjiang province, in northeast China, where the proportion of MDR-TB (12.1\%) was estimated to be one of the three highest in the country, much higher than the national average of $8.32 \%$ in $2007 .{ }^{12}$ Unfortunately, there is a conspicuous gap in research into MDR-TB between high and low prevalence regions in China. ${ }^{7}$ Despite some studies carried out in low-prevalence regions, ${ }^{13}{ }^{14}$ the factors influencing MDR-TB in high-prevalence regions have rarely been investigated. This study aimed to identify the factors contributing to the high prevalence of MDR-TB in Heilongjiang province.

\section{METHODS}

\section{Study population}

Heilongiiang has a population of 38.1 million and is divided into 113 counties (or districts). Thirty 
counties were selected at random as surveillance sites of drug resistance in $\mathrm{TB} .{ }^{15}$ The surveillance covered all public and private facilities in the selected counties and used the guidelines developed by the WHO and the International Union against Tuberculosis and Lung Disease. ${ }^{16}$ From January to December 2004, MDR-TB was detected in 30 surveillance sites through drug susceptibility testing (DST). At each surveillance site, the first 56 new patients with $\mathrm{TB}$ and all of the retreatment patients who sought services were monitored for MDR-TB. A new patient was defined as a patient with TB who had never been treated for $\mathrm{TB}$ or who had received anti-TB drugs treatment for $<1$ month. A retreatment patient was defined as a patient with TB who had a prior history of treatment with anti-TB drugs for $>1$ month. $^{17}$

\section{Drug susceptibility testing}

Culture and DST is not routinely done in new patients with TB in Heilongjiang. In this study, a patient whose sputum sample was smear positive for acid-fast bacilli was defined as having sputum smear positive pulmonary TB. A positive sputum smear for Gram stain was rated from $1+$ to $4+$, with a higher number indicating a stronger presence of smear-positive stain. The culture positive samples were sent to the provincial dispensary for DST.

The Heilongjiang reference laboratory performed DST with the sputum cultures from the study participants. DST included four first-line antibiotics: rifampin, isoniazid, ethambutol and streptomycin. Validation and quality assurance of the test results were provided by the Chinese National Reference Laboratory and the WHO Supranational Reference Laboratory. All DST strictly followed the procedure and methods set out by WHO and the International Union against Tuberculosis and Lung Disease. ${ }^{16}$

\section{Questionnaire survey}

Patients were invited to complete a questionnaire administered by well trained medical workers through face-to-face interviews when the patients attended the TB services. Data collected included demographical characteristics of the patients, clinical features of TB and outcomes of previous TB treatments.

\section{In-depth interview}

The interviewees were purposively selected based on their roles and experience in TB control. Nine retreatment patients, four physicians and five health administrative officials and TB project managers completed the interviews. Patients with TB were asked to answer when, where and why they had received TB services, how they were treated and how they had managed their medications. The managers and officials were asked to answer questions in relation to the arrangements of treatment regimens and compliance of providers and patients. The physicians were asked to answer questions about their experiences and problems in treating patients with TB.

The interviews were digitally recorded, transcribed and thematically coded. The final sample size was determined by saturation of information when no new categories emerged.

\section{Data analysis}

\section{Quantitative analysis}

Demographical and clinical characteristics and service patterns of the patients with MDR-TB were compared with those of patients without MDR-TB. Because new patients might have different influencing factors compared with retreatment patients, two regression models were constructed for new cases and retreatment cases, respectively. Independent variables tested in the regression analysis included gender, age, working status, delay in initiating TB treatment (delay between onset of symptoms and presenting for treatment in the most recent episode), cavitary diseases, TB in organs other than lungs and diabetes. For the retreatment patients, variables about previous TB treatment were also included, such as the number of previous treatment regimens (an episode of $\mathrm{TB}$ refers to the period from onset of symptoms to end of treatment; a treatment regimen refers to all prescribed treatments during an episode of $\mathrm{TB})$, service providers of the last treatment, and length of treatment with rifampin and isoniazid (accumulated days of previous treatment with rifampin and isoniazid). All variables were entered into the regression models, except for those that exhibited strong collinearity.

The regression models were determined by intra-class correlation coefficients (ICCs) of the independent variables. A traditional logistic regression model was applied to the retreatment cases for which the ICC was not significant. Multilevel models were applied to the entire sample and the subsample of new cases because their ICCs were significant. Multilevel models considered individual level variables and group level variables, ${ }^{18}$ which helps to avoid underestimating the standard errors, therefore avoiding overestimation of the significance of the influencing factors. ${ }^{19}$

All analyses were performed using SAS V.9.1.

\section{Qualitative analysis}

The interview data were analysed thematically. The coding framework was developed inductively from the data. The initial coding used open coding (codes derived directly from the data) and theoretical coding. The initial codes were then refined to produce a smaller set of themes. The coding framework was subject to continuing iterative revision during the course of analysis.

\section{Ethics approval}

The study protocol was reviewed and approved by the Research Ethics Committee of Harbin Medical University.

\section{RESULTS}

\section{Characteristics of the study population}

A total of 2144 patients were recruited for sputum cultures, 1995 of whom had positive sputum cultures and underwent DST. A $100 \%$ response rate was achieved. Missing data accounted for $<5 \%$ in any single questionnaire item. The 1995 study participants had a median age of 43 years and $69.4 \%$ were men. MDR was detected in 241 of the study participants $(12.1 \%$, 95\% CI $10.7 \%$ to $13.5 \%$ ). More than $58 \%$ of the patients were diagnosed and treated for TB within 60 days of occurrence of symptoms. About $65 \%$ of the sputum smear samples were rated as $4+$ for Gram stain; $37.7 \%$ of patients had at least one pulmonary cavity; $1.7 \%$ had TB in organs other than the lungs; $4.0 \%$ had diabetes mellitus; $0.1 \%$ had pneumoconiosis; and $0.1 \%$ had mental illness (table 1).

There were 421 retreatment patients, which comprised $21.1 \%$ of the total study sample. The retreatment patients had greater prevalence of MDR-TB (30.4\%, 95\% CI $26.0 \%$ to $34.8 \%)$ than the new patients $(7.2 \%, 95 \%$ CI $5.9 \%$ to $8.5 \%)(p<0.001)$. The retreatment patients had received an average of 1.49 treatment regimens in the past. More than $72 \%$ had received $\leq 180$ accumulative days of treatment with isoniazid and rifampin in the past. 
Table 1 Demographical and clinical characteristics of the questionnaire respondents

\begin{tabular}{|c|c|c|c|}
\hline Characteristics & $\begin{array}{l}\text { MDR-TB } \\
(n=241)\end{array}$ & $\begin{array}{l}\text { Non-MDR-TB } \\
(\mathrm{n}=1754)\end{array}$ & p ( $\chi^{2}$ test $)$ \\
\hline \multicolumn{4}{|l|}{ Sex } \\
\hline Men & $171(71.0)$ & $1214(69.2)$ & 0.582 \\
\hline Women & $70(29.0)$ & $540(30.8)$ & \\
\hline Age, median years (range) & $43(20-83)$ & $44(16-92)$ & \\
\hline$<45$ years & $140(58.1)$ & $909(51.8)$ & 0.068 \\
\hline$\geq 45$ years & $101(41.9)$ & $845(48.2)$ & \\
\hline \multicolumn{4}{|l|}{ Working status } \\
\hline Working & $185(76.8)$ & $1192(68.0)$ & 0.006 \\
\hline Not working & $56(23.2)$ & $562(32.0)$ & \\
\hline \multicolumn{4}{|l|}{ Treated for TB in the past } \\
\hline Yes & $128(53.1)$ & $293(16.7)$ & $<0.001$ \\
\hline No & $113(46.9)$ & $1461(83.3)$ & \\
\hline \multicolumn{4}{|c|}{ Delay in initiating TB treatment } \\
\hline$\leq 60$ days & $114(47.3)$ & $1045(59.6)$ & $<0.001$ \\
\hline$>60$ days & $127(52.7)$ & $709(40.4)$ & \\
\hline \multicolumn{4}{|l|}{ Cavitary disease } \\
\hline Yes & $100(41.5)$ & $645(36.8)$ & \\
\hline No & $140(58.1)$ & $1090(62.1)$ & \\
\hline Missing/unknown & $1(0.4)$ & $19(1.1)$ & \\
\hline \multicolumn{4}{|l|}{ TB in organs other than lungs } \\
\hline Yes & $8(3.3)$ & $25(1.4)$ & \\
\hline No & $233(96.7)$ & $1726(98.4)$ & \\
\hline Missing/unknown & 0 & $3(0.2)$ & \\
\hline \multicolumn{4}{|l|}{ Complicating health conditions } \\
\hline No & $227(94.2)$ & $1630(93.0)$ & \\
\hline Diabetes mellitus & $10(4.1)$ & $69(3.9)$ & \\
\hline Pneumoconiosis & $1(0.4)$ & $1(0.1)$ & \\
\hline Mental illness & $1(0.4)$ & $2(0.1)$ & \\
\hline Lung infection & $1(0.4)$ & $35(2.0)$ & \\
\hline Others & $1(0.4)$ & $16(0.9)$ & \\
\hline
\end{tabular}

Data are numbers (\%) of patients unless otherwise indicated.

MDR-TB, multidrug resistant TB.

The outcome of the last treatment was not satisfactory, with only $27.8 \%$ of patients being cured or completing treatment and in $21.4 \%$ the outcome was unknown. More than $42 \%$ did not complete the treatment regimens (table 2). The patients treated in general hospitals or other clinics had a higher percentage of incomplete treatment $(50.7 \%)$ than those treated in $\mathrm{TB}$ dispensaries $(29.9 \%, \mathrm{p}<0.001)$.

\section{Factors associated with MDR-TB}

With the 1972 cases (missing data excluded) entered into the multilevel model, history of previous treatment became the strongest association with MDR-TB. The retreatment patients were 5.48 times (95\% CI 4.04 to 7.44 ) more likely to have MDRTB than the newly diagnosed patients (table 3 ). Other influencing factors included age (adjusted OR (AOR) for being younger than 45 years $=1.75,95 \%$ CI 1.29 to 2.37 ) and delay in initiating TB treatment (AOR for $>60$ days $=1.43$, 95\% CI 1.05 to 1.94 ).

The multilevel model with the 1554 new cases (missing data excluded) showed that a younger age ( $A O R=1.72,95 \% \mathrm{CI} 1.13$ to 2.61 ) remained statistically significant (table 4 ).

The logistic regression model with the 418 retreatment cases (missing data excluded) showed that the pattern of TB services had a significant impact on the development of MDR-TB. Patients with $>180$ accumulative days of treatment with rifampin and isoniazid were 4.82 times (95\% CI 2.97 to 7.81 ) more likely to develop MDR-TB than those with $<180$ days of treatments. Further analysis showed that, of the patients with
Table 2 Patterns of tuberculosis (TB)-related clinical services received by the 421 retreatment patients

\begin{tabular}{lc}
\hline Characteristics & Value \\
\hline Numbers of previous treatments, median (range) & $1(1-9)$ \\
1 & $294(69.8)$ \\
$>1$ & $127(30.2)$ \\
Provider of last treatment & \\
TB dispensary/TB hospital & $202(48.0)$ \\
General hospital/others & $219(52.0)$ \\
Outcome of last treatment & \\
Unknown & $90(21.4)$ \\
Cured & $100(23.8)$ \\
Treatment completed & $17(4.0)$ \\
Failure & $25(5.9)$ \\
Treatment uncompleted & $178(42.3)$ \\
Transferred out & $9(2.1)$ \\
Data missing & $2(0.5)$ \\
Length of treatment with rifampin and isoniazid $(\mathrm{n}=420)$ & \\
$\leq 90$ days & $151(36.0)$ \\
$91 \sim 180$ days & $152(36.2)$ \\
$181 \sim 240$ days & $41(9.8)$ \\
$>240$ days & $76(18.1)$ \\
\hline
\end{tabular}

Data are numbers (\%) of patients unless otherwise indicated.

$>180$ accumulative days of treatment with isoniazid and rifampin, those who had undergone two or more regimens were 1.82 times more likely to develop MDR-TB than those who had undergone only one regimen $(66.7 \%$ vs $47.6 \%)$. Delay in initiating $\mathrm{TB}$ treatment was the second strongest association with

Table 3 Multivariable analysis for estimation of prevalence of MDR-TB in the entire sample* $(n=1972)$

\begin{tabular}{|c|c|c|c|}
\hline Variable & $\begin{array}{l}\text { Prevalence of } \\
\text { MDR-TB (\%) }\end{array}$ & AOR $(95 \%$ Cl) & p Multilevel \\
\hline \multicolumn{4}{|l|}{ Sex } \\
\hline Men & 12.3 & $1.06(0.77$ to 1.47$)$ & \multirow[t]{2}{*}{0.714} \\
\hline Women & 11.5 & 1 (reference) & \\
\hline \multicolumn{4}{|l|}{ Age } \\
\hline$<45$ years & 13.3 & 1.75 (1.29 to 2.37$)$ & \multirow[t]{2}{*}{$<0.001$} \\
\hline$\geq 45$ years & 10.7 & 1 (reference) & \\
\hline \multicolumn{4}{|l|}{ Not working } \\
\hline Yes & 9.1 & 0.76 (0.53 to 1.09$)$ & \multirow[t]{2}{*}{0.136} \\
\hline No & 13.4 & 1 (reference) & \\
\hline \multicolumn{4}{|c|}{ Delay in initiating TB treatment } \\
\hline$>60$ days & 15.2 & $1.43(1.05$ to 1.94$)$ & \multirow[t]{2}{*}{0.024} \\
\hline$\leq 60$ days & 9.8 & 1 (reference) & \\
\hline \multicolumn{4}{|c|}{ Treated for TB in the past } \\
\hline Yes & 30.4 & 5.48 (4.04 to 7.44$)$ & \multirow[t]{2}{*}{$<0.001$} \\
\hline No & 7.2 & 1 (reference) & \\
\hline \multicolumn{4}{|c|}{ Cavitary disease } \\
\hline Yes & 13.4 & 1.21 (0.89 to 1.66$)$ & \multirow[t]{2}{*}{0.222} \\
\hline No & 11.4 & 1 (reference) & \\
\hline \multicolumn{4}{|c|}{ TB in organs other than lungs } \\
\hline Yes & 24.2 & $2.23(0.88$ to 5.66$)$ & \multirow[t]{2}{*}{0.092} \\
\hline No & 11.9 & 1 (reference) & \\
\hline \multicolumn{4}{|c|}{ Diabetes mellitus } \\
\hline Yes & 12.7 & $1.39(0.66$ to 2.90$)$ & \multirow[t]{3}{*}{0.383} \\
\hline No & 12.1 & 1 (reference) & \\
\hline ICC & & 0.084 & \\
\hline
\end{tabular}

The associations between multidrug-resistant tuberculosis (MDR-TB) and independent variables are expressed as adjusted OR (AOR) with $95 \% \mathrm{CI}$.

*Multilevel model was fitted using SAS PROC GLIMMIXED.

ICC, intra-class correlation coefficient. 
Table 4 Multivariable analysis for estimation of prevalence of MDR-TB in the new cases* $(n=1554)$ and retreatment cases $\dagger(n=418)$

\begin{tabular}{|c|c|c|c|c|c|c|}
\hline \multirow[b]{2}{*}{ Variable } & \multicolumn{3}{|c|}{ New cases $(n=1554)$} & \multicolumn{3}{|c|}{ Retreatment cases $(n=418)$} \\
\hline & $\%$ of MDR-TB & AOR $(95 \% \mathrm{Cl})$ & p Multilevel & $\%$ of MDR-TB & AOR $(95 \% \mathrm{CI})$ & $\mathbf{p}$ \\
\hline \multicolumn{7}{|l|}{ Sex } \\
\hline Men & 7.9 & 1.45 (0.91 to 2.31$)$ & 0.114 & 28.1 & 0.72 (0.43 to 1.21$)$ & 0.213 \\
\hline Women & 5.5 & 1 (reference) & & 36.1 & 1 (reference) & \\
\hline \multicolumn{7}{|l|}{ Age } \\
\hline$<45$ years & 8.5 & $1.72(1.13$ to 2.61$)$ & 0.012 & 36.9 & $1.44(0.91$ to 2.30$)$ & 0.122 \\
\hline$\geq 45$ years & 5.5 & 1 (reference) & & 25.6 & 1 (reference) & \\
\hline Yes & 5.0 & 0.71 (0.43 to 1.18 ) & 0.184 & 27.4 & 0.68 (0.39 to 1.16$)$ & 0.158 \\
\hline No & 8.2 & 1 (reference) & & 31.5 & 1 (reference) & \\
\hline \multicolumn{7}{|c|}{ Delay in initiating TB treatment } \\
\hline$>60$ days & 6.8 & 1.09 (0.71 to 1.66$)$ & 0.692 & 38.6 & 2.64 (1.61 to 4.30$)$ & $<0.001$ \\
\hline$\leq 60$ days & 7.4 & 1 (reference) & & 21.4 & 1 (reference) & \\
\hline \multicolumn{7}{|c|}{ Cavitary disease } \\
\hline No & 7.1 & 1 (reference) & & 30.1 & 1 (reference) & \\
\hline \multicolumn{7}{|c|}{ Diabetes mellitus } \\
\hline Yes & 6.7 & $1.39(0.48$ to 4.03$)$ & 0.544 & 31.6 & 1.45 (0.49 to 4.32$)$ & 0.507 \\
\hline No & 7.2 & 1 (reference) & & 30.3 & 1 (reference) & \\
\hline \multicolumn{7}{|c|}{ Provider of last treatment } \\
\hline TB dispensa & & & & 35.6 & 1.84 (1.14 to 2.97$)$ & 0.012 \\
\hline General hos & & & & 25.6 & 1 (reference) & \\
\hline \multicolumn{7}{|c|}{ Length of treatment with rifampin and isoniazid } \\
\hline$>180$ days & & & & 56.0 & 4.82 (2.97 to 7.81$)$ & $<0.001$ \\
\hline$\leq 180$ days & & & & 20.7 & 1 (reference) & \\
\hline ICC & & 0.113 & & & 0.023 & \\
\hline
\end{tabular}

The associations between multidrug resistant tuberculosis (MDR-TB) and independent variables were expressed as adjusted $0 \mathrm{R}$ (AOR) with 95\% $\mathrm{Cl}$.

*Two-level multilevel model was fitted using SAS PROC GLIMMIXED.

†Binominal regression model was fitted using PROC LOGISTIC.

ICC, intra-class correlation coefficient.

MDR-TB. Patients diagnosed and treated after 60 days from the onset of symptoms were 2.64 times (95\% CI 1.61 to 4.30 ) more likely to have MDR-TB than those treated more promptly. The AOR of MDR-TB for TB dispensaries was 1.84 (95\% CI 1.14 to 2.97) compared with general hospitals (table 4).

\section{Reasons for intermittent TB treatment}

The findings of the survey were supported by the interviews with patients, health managers and physicians.

The patients confirmed that dropout from TB treatment regimens was common. The top three reasons for failure to complete the TB treatment regimens were financial burden, poor knowledge and treatment side effects. TB treatment was usually free at the designated TB dispensaries; however, such a free treatment policy seldom extended to general hospitals and clinics, where $45.1 \%$ of the retreatment patients obtained their last treatment. Even for those who received free treatment at TB dispensaries, patients were out of pocket for extra costs for drugs to combat side effects of the treatment and second-line drugs when needed. Moreover, due to insufficient knowledge about the serious consequences of interrupted and intermittent treatment, many patients tended to give up treatment when symptoms disappeared, side effects kicked in or social support was difficult to obtain.

Irrational prescriptions, poorly formulated medications, insufficient dosage and length of treatment, and inadequate administration of drugs were believed to be the most common practices that might promote drug resistance. The physicians acknowledged inappropriate treatment of TB occurred as a result of a lack of proper training and poor compliance with TB treatment guidelines.

Although there is a policy requirement for $\mathrm{TB}$ to be treated in designated TB dispensaries and TB hospitals, an effective referral system is missing. Consumers have the freedom to seek medical services from any providers. Unfortunately, the directly observed treatment, short-course (DOTS) strategy had not been strictly followed by hospitals. This was believed to have exacerbated the interrupted and intermittent TB treatment. In addition, a lack of targeted training and supervision, ignorance of standard $\mathrm{TB}$ treatment guidelines and unsatisfactory infection control in some hospitals also contributed to the development of MDR-TB. The healthcare managers pointed out that a lack of relevant legislation and regulations, limited public funding and poor cooperation among healthcare providers had formed a bottleneck for the continuing and coordinated care of patients with TB.

Factors associated with the high prevalence of MDR-TB in Heilongjiang are illustrated in figure 1, which combines the findings from the quantitative and qualitative analysis. The influencing factors are grouped into three categories: environment, host and pathogen. The environmental category, which includes the policy and service delivery system and public attitudes towards TB, might have a significant impact on the behaviour of patients with TB. System failure and poor performance of healthcare providers were found to lead to potential delays in $\mathrm{TB}$ treatment and exacerbate poor compliance of 
Figure 1 Factors associated with multidrug-resistant tuberculosis (MDR-TB).

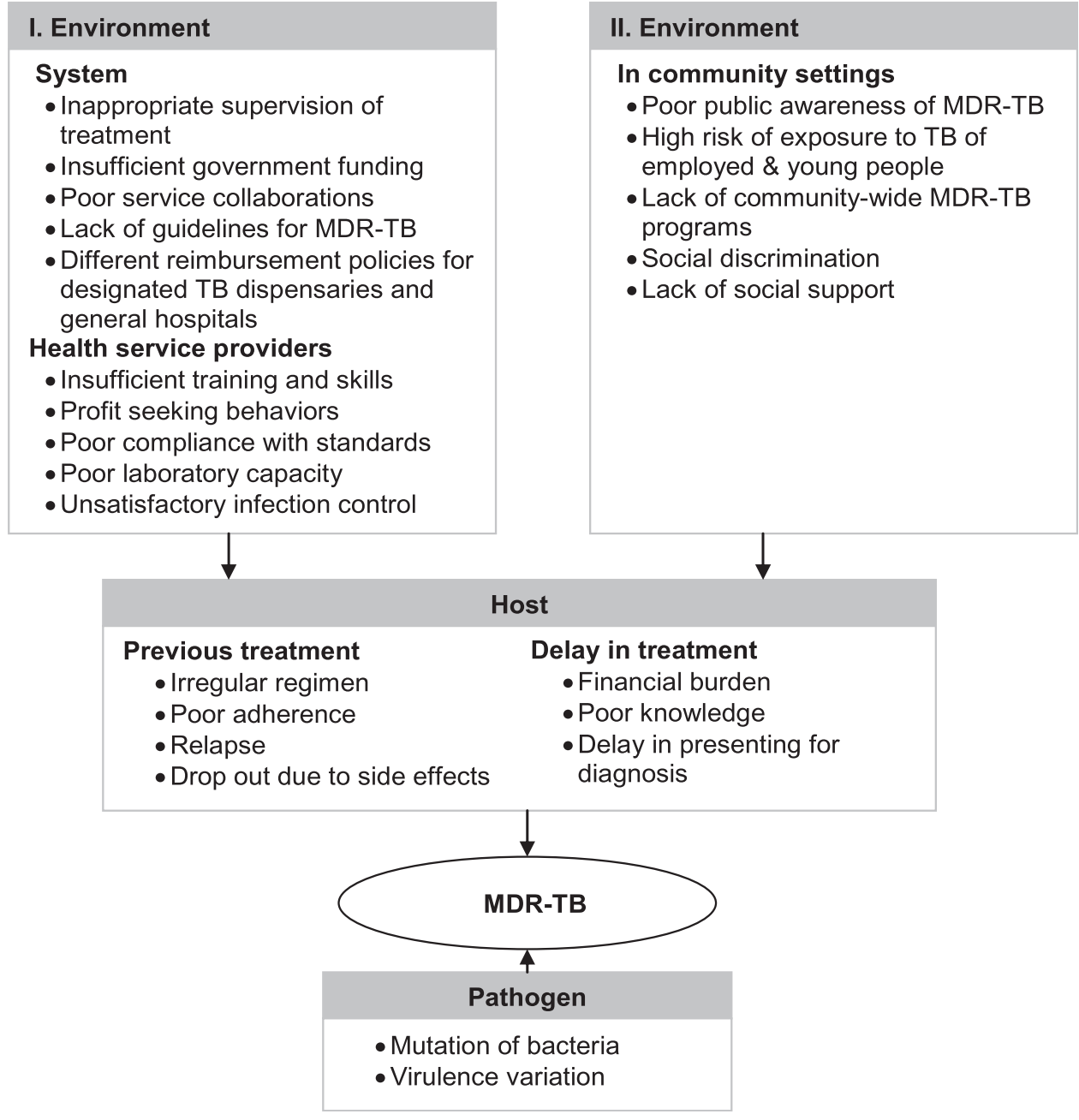

patients undergoing TB treatment. Meanwhile, young and financially disadvantaged patients and those with poor knowledge about TB were found to be likely to develop MDR-TB.

\section{DISCUSSION}

Besides the major biological factors leading to the prevalence of MDR-TB, such as the fitness of drug-resistant mycobacterium $\mathrm{TB}$ strains, ${ }^{20}$ mutation ability ${ }^{21}$ and virulence variation, ${ }^{22} \mathrm{~TB}-$ related service patterns have a significant impact on the development of MDR-TB. Not surprisingly, retreatment was identified as the strongest association with MDR-TB, which is consistent with previous studies. ${ }^{3} 911$ More than $30 \%$ of retreatment patients were identified with MDR-TB in our study. Poor outcomes of previous treatments and the relapse of $\mathrm{TB}$ might have contributed to the high prevalence of MDR-TB. China has a very high relapse rate of $\mathrm{TB}$. The TB relapse rate of patients in Shanghai was reported to be $61.5 \%{ }^{23}$ This would inevitably lead to prolonged treatment and increase the chance of developing MDR-TB. ${ }^{24}$

Patients treated with isoniazid and rifampin for $>180$ days were more likely to have MDR-TB than other patients. A lack of qualified DST is perhaps one of the potential causes of MDR-TB. As in many other developing countries, many patients with TB in China are treated empirically without DST. Failure to respond to first-line drugs is sometimes the only way to identify drug resistance, ${ }^{3} 25$ which requires observations for an extensive period of time. Lacking an effective and rapid detection system for drug resistance, many developing countries offer standardised treatment as an expedient for all patients regardless of their drug resistance profile. ${ }^{26}$

Delay in initiating treatment prolongs the time that patients carry the TB bacteria and allows the disease to progress without proper intervention. ${ }^{27}$ Reasons for the delay might be due to the patients or the healthcare providers. Patients might not seek medical services because of poor knowledge about TB, mild symptoms, financial burden and poor accessibility to TB dispensaries. Chen's study found that $26.4 \%$ of patients with TB delayed their treatment, mostly because of mild symptoms $(54.4 \%)$ and financial burden $(33.3 \%) .^{28}$ Although the Chinese government has a policy of free diagnosis and treatment of TB, this policy applies only to the first two treatment regimens except ancillary drugs. Some physicians, especially those who work in rural clinics, do not have sufficient knowledge of $\mathrm{TB}$, which often results in delays in diagnosis and treatment. ${ }^{29}$

Some patients might give up their treatment due to side effects. A systematic review found that $12.62 \%$ of patients with $\mathrm{TB}$ in China have side effects from the treatment drugs. ${ }^{30}$ In addition, social stigma and a lack of social support have created barriers preventing patients from obtaining and maintaining treatment. A lack of time to seek medical services has often been used as an excuse by patients for delaying services for fear of social discrimination. This is particularly true in younger patients. ${ }^{3} 13$ Yan and Jiang's study found that young people 
tended to postpone their treatment for fear of discrimination, dismissal and difficulty in finding a partner and getting married. $^{31}$

Successful control of MDR-TB depends on collaboration between multiple service providers. According to the policy of TB control, patients have been required to obtain free treatment at designated TB dispensaries or TB hospitals since 1995. However, this study showed that as many as $51.8 \%$ of retreatment patients received their last treatment at general hospitals and clinics. Such hospitals and clinics compete to attract more patients to earn extra profits. ${ }^{32}$ Even with a confirmed diagnosis of $\mathrm{TB}$, they are reluctant to refer patients with $\mathrm{TB}$ to the designated TB dispensaries. Patients whose condition failed to respond to previous treatment were likely to be referred to designated TB dispensaries as the last TB treatment provider, which may result in them developing MDR-TB.

The DOTS strategy has been proven to be one of the most effective strategies for managing TB. DOTS coverage in China rapidly increased from $68 \%$ in 2000 to $100 \%$ in $2005 .{ }^{33}$ However, our study showed that loopholes exist in the healthcare system, which undermine the effective detection and management of TB. Patients with TB treated at general hospitals and clinics were found to be rarely exposed to DOTS supervision. The healthcare managers interviewed believed that irregular and incomplete treatment has contributed to the poor outcome of patients with TB. Evidence from other studies also supports this argument. ${ }^{34}$ As a result of an inefficient system, $<50 \%$ of TB cases were detected in 2004 in China (74\% in Heilongjiang) despite an overall $85 \%$ success rate in treatment. ${ }^{33}$ The DOTS strategy in China has been implemented by the designated TB dispensaries. Despite a reported 100\% DOTS coverage, the outcome of the strategy is not satisfactory. Our study showed that $29.9 \%$ of patients treated at TB dispensaries did not complete their recent treatment regimen.

Our study has several limitations. First, data about people's contact with TB or MDR-TB were not available. TB contact history has been found to be associated with drug resistance in other studies. ${ }^{13}$ Second, DST of second-line drugs was not performed. As a result, extensive drug resistant TB could not be included in the analysis. Third, due to a very low HIV prevalence $(0.5 / 100000)$ in Heilongjiang in $2004,{ }^{35}$ the association between $\mathrm{HIV}$ infection and MDR-TB was not analysed. Despite the limitations, this study provides important findings for better planning of TB management in regions where MDR-TB prevalence is high.

In conclusion, inappropriate treatment of $\mathrm{TB}$ is the most important factor contributing to the development of MDR-TB. There is an urgent need to increase people's awareness of the threat of MDR-TB. Early detection, diagnosis and treatment of patients with TB should become a priority in the control of MDR-TB. Strong commitment and collaboration among health organisations, continuing and coordinated care for patients with $\mathrm{TB}$, together with greater compliance by service providers and patients with TB treatment guidelines are key to success in the battle against MDR-TB.

Acknowledgements The authors thank the staff of Heilongjiang TB Control Center/ National TB Control Center for their support and help with this study.

Contributors $\mathrm{QW}, \mathrm{LG}, \mathrm{YX}$ and $\mathrm{YH}$ designed and planned this study; $Y X, L G, H S, X Y$, $\mathrm{FL}, \mathrm{HL}$ and $\mathrm{HF}$ undertook the fieldwork and data collection; $\mathrm{LL}, \mathrm{NN}$, LH and YC performed statistical analysis; LL, $\mathrm{OW}, \mathrm{YH}$ and $\mathrm{CL}$ integrated data analysis and wrote a first draft; $\mathrm{OW}, \mathrm{LL}$ and $\mathrm{CL}$ revised the paper.

Funding Funding was provided in part by the China Medical Board and the China Global Fund TB Programme 08-929.
Competing interests None.

Patient consent Obtained.

Provenance and peer review Not commissioned; externally peer reviewed.

\section{REFERENCES}

1. Gandhi NR, Nunn P, Dheda K, et al. Multidrug-resistant and extensively drugresistant tuberculosis: a threat to global control of tuberculosis. Lancet 2010;375:1830-43

2. Ormerod LP. Multidrug-resistant tuberculosis (MDR-TB): epidemiology, prevention and treatment. Br Med Bull 2005;73-74:17-24.

3. World Health Organization. Multidrug and Extensively Drug-resistant TB (M/XDRTB): 2010 Global Report on Surveillance and Response. Switzerland, Geneva: World Health Organization, 2010.

4. World Health Organization. Global Tuberculosis Control: Epidemiology, Strategy, Financing. Switzerland, Geneva: World Health Organization, 2009.

5. World Health Organization. A Ministerial Meeting of High M/XDR-TB Burden Countries. Geneva, Switzerland: World Health Organisation, 2009

6. Wang L, Liu J, Chin DP. Progress in tuberculosis control and the evolving publichealth system in China. Lancet 2007;369:691-6.

7. Yang $\mathbf{X Y}$, Li YP, Mei YW, et al. Time and spatial distribution of multidrug-resistant tuberculosis among Chinese people, 1981-2006: a systematic review. Int J Infect Dis 2010;14:e828-37.

8. Lomtadze N, Aspindzelashvili R, Janjgava $\mathrm{M}$, et al. Prevalence and risk factors for multidrug-resistant tuberculosis in the Republic of Georgia: a population-based study. Int J Tuberc Lung Dis 2009;13:68-73.

9. Moniruzzaman A, Elwood RK, Schulzer M, et al. A population-based study of risk factors for drug-resistant TB in British Columbia. Int J Tuberc lung Dis 2006:10:631-8.

10. Suarez-Garcia I, Rodriguez-Blanco A, Vidal-Perez JL, et al. Risk factors for multidrug-resistant tuberculosis in a tuberculosis unit in Madrid, Spain. Eur J Clin Microbiol Infect Dis 2009;28:325-30.

11. Faustini A, Hall AJ, Perucci CA. Risk factors for multidrug resistant tuberculosis in Europe: a systematic review. Thorax 2006:61:158-63.

12. He GX, Zhao $\mathrm{YL}$, Jiang $\mathrm{GL}$, et al. Prevalence of tuberculosis drug resistance in 10 provinces of China. BMC Infect Dis 2008;8:166.

13. Shen X, DeRiemer K, Yuan ZA, et al. Drug-resistant tuberculosis in Shanghai, China, 2000-2006: prevalence, trends and risk factors. Int J Tuberc Lung Dis 2009;13:253-9.

14. Chen J, Li NX, Wan KL, et al. Analysis on risk factors of drug resistance for tuberculosis in Sichuan and Anhui province (In Chinese). J Sichuan Univ (Med Sci Edi) 2007;38:135-7.

15. Xie YG, Li FB, Yan XL. WHO TB drug resistance survey in Heilongjiang province. Chin $J$ Antituberculosis Association 2008;30:395-8.

16. World Health Organization. Guidelines for Surveillance of Drug Resistance in Tuberculosis. Geneva, Switzerland: World Health Organization, 1997.

17. World Health Organization. Treatment of Tuberculosis: Guidelines for National Programmes. 3rd edn. Geneva: World Health Organization, 2003.

18. Merlo J. Multilevel analytical approaches in social epidemiology: measures of health variation compared with traditional measures of association. J Epidemiol Community Health 2003:57:550-2.

19. Hox JJ. Multilevel Analysis, Techniques and Application. Mahwah, NJ: Lawrence Erlbaum Associates, 2002.

20. Cohen T, Sommers B, Murray M. The effect of drug resistance on the fitness of mycobacterium tuberculosis. Lancet Infect Dis 2003;3:13-21.

21. Ano H, Matsumoto T, Suetake T, et al. Relationship between the isoniazid-resistant mutation katGS315T and the prevalence of MDR-/XDR-TB in Osaka, Japan. Int $J$ Tuberc Lung Dis 2008;12:1300-5.

22. Marquina-Castillo B, Garcia-Garcia L, Ponce-de-León A, et al. Virulence, immunopathology and transmissibility of selected strains of Mycobacterium tuberculosis in a murine model. Immunology 2009;128:123-33.

23. Shen G, Xue Z, Shen $X$, et al. The study recurrent tuberculosis and exogenous reinfection, Shanghai, China. Emerg Infect Dis 2006;12:1776-8.

24. Jeon CY, Hwang SH, Min JH, et al. Extensively drug-resistant tuberculosis in South Korea: risk factors and treatment outcomes among patients at a tertiary referral hospital. Clin Infect Dis 2008;46:42-9.

25. Seung KJ. Does my patient have multidrug-resistant tuberculosis? Clin Infect Dis 2010:51:379-80

26. Laniado-Laborin R. Multidrug-resistant tuberculosis: standardized or individualized treatment? The question has already been answered. Expert Rev Respir Med 2010;4:143-6.

27. Nic Fhogartaigh CJ, Vargas-Prada S, Huancare V, et al. Physician-initiated courtesy MODS testing for TB and MDR-TB diagnosis and patient management. Int $\mathrm{J}$ Tuberc Lung Dis 2008;12:555-60.

28. Chen $\mathbf{0}$, Zheng J, Wu N. Analysis on the correlated factors of delay in tuberculosis diagnosis and treatment in Fujian province. Chin J Antituberculosis Association 2005:27:374-9.

29. Wang W, Jiang 0 , Abdullah AS, et al. Barriers in accessing to tuberculosis care among non-residents in Shanghai: a descriptive study of delays in diagnosis. Eur $J$ Public Health 2007;17:419-23. 
30. Xia Y, Zhan S. Systematic review of anti-tuberculosis drug induced adverse reactions in China (In Chinese). Chin J Tuberc Respir Dis 2007;30:419-23.

31. Yan F, Jiang 0. Current Situation and Strategy for Tuberculosis Control in China, Social Assessment Study in Four Provinces. Fudan University, Shanghai, doctor dissertation, 2007.

32. Meng 0, Li R, Cheng G, et al. Provision and financial burden of TB services in a financially decentralized system: a case study from Shandong, China. Int J Health Plann Manage 2004;19(Suppl 1):S45-62.
33. World Health Organization. Tuberculosis Control in the Western Pacific Region: 2009 Report. Geneva: World Health Organization, 2009.

34. Tang S, Squire SB. What lessons can be drawn from tuberculosis (TB) control in China in the 1990s? An analysis from a health system perspective. Health Policy 2005; 72:93-104

35. Zhang J, Luo J, Wang L, et al. The analysis of HIV/AIDS epidemic in Heilongjiang province from 1993 to 2004. Chin J AIDS STD 2005;11:264-6.

\section{Journal club}

\section{Exhale airway bypass stents for emphysema}

Existing treatment options for severe homogenous emphysema are limited, with dynamic airways collapse and gas trapping minimising the benefits of drug therapy. There has been interest in therapies that address these features more directly. The bronchoscopic lung volume reduction with Exhale airway stents for emphysema trial study group recently reported their findings in The Lancet. The group undertook a multicentre, double-blind, randomised, sham-controlled trial at 38 specialist respiratory centres worldwide. Three hundred and fifteen patients with severe homogenous emphysema were randomised to have sham bronchoscopy $(\mathrm{n}=107)$, or up to six Exhale paclitaxel-eluting stents implanted bronchoscopically $(\mathrm{n}=212)$. These stents create artificial airways connecting central airways with the emphysematous lung parenchyma and serve as conduits which allow trapped gas to escape thereby reducing both static and dynamic hyperinflation. Unlike lung volume reduction surgery or lung volume reduction using endobronchial valves, this approach does not involve loss of functioning lung tissue by resection or atelectasis.

On day 1 following the procedure, a difference in the reduction of the residual volume of $26 \%$ and an increase in the forced vital capacity of $27 \%$ were seen between the two groups. Significant differences were also seen in CT-measured lobar volumes and forced expiratory volume in one second between the groups. Unfortunately, these benefits were not maintained, with lung function measures and CT-measured lobar volumes returning to baseline by 3 months following treatment. This is primarily due to airway bypass stent occlusion by mucus, granulation tissue or stent expectoration.

This study serves as a 'proof of principle' that bronchoscopic airway bypass can be a successful approach to treat lung hyperinflation and gas trapping in homogenous emphysema. However, novel methods of maintaining stent patency need to be developed before moving forward with this approach. Valuable lessons can be learnt from this trial's robust and novel study design, which has set a new benchmark for future trials evaluating bronchoscopic and clinical procedures or novel medical devices. This study demonstrates that it is possible to undertake randomised, double-blind, sham-controlled trials of interventional procedures safely in this high-risk patient population.

Shah PL, Slebos DJ, Cardoso PF, et al; EASE trial study group. Bronchoscopic lung-volume reduction with Exhale airway stents for emphysema (EASE trial): randomised, sham-controlled, multicentre trial. Lancet 2011;378:997-1005.

\section{Zaid Zoumot}

Correspondence to Dr Zaid Zoumot, Interventional Bronchoscopy Clinical Research Fellow, The Royal Brompton Hospital, Sydney Street, London SW3 6NP, UK; zzoumot@doctors.org.uk

Provenance and peer review Not commissioned; internally peer reviewed.

Published Online First 6 December 2012

Thorax 2012;67:638. doi:10.1136/thoraxjnl-2011-201372 